\title{
BDE-47 and BDE-209 inhibit proliferation of Neuro-2a cells via inducing G1-phase arrest
}

Hongmei Chen, Xuexi Tang, Bin Zhou, Ningning Xu, Zhongyuan Zhou, Kuan Fang, You Wang ${ }^{*}$

Department of Marine Ecology, College of Marine Life Sciences, Ocean University of China, Qingdao 266003, China. E-mail: wangyou@ ouc.edu.cn

\begin{abstract}
Cell proliferation is closely related to cell cycle which is strictly regulated by genes and regulatory proteins. In the present study, we comparatively analyzed the toxic effects of BDE47 and BDE-209 on cell proliferation of Neuro-2a cells, and the possible mechanism was discussed. The results indicated that BDE-47 significantly inhibited the cell proliferation and the cell cycle were arrest at G1 phase, while BDE-209 had little effects on either cell proliferation or cell cycle. qRT-PCR and Western blot assay presented that BDE-47 upregulated the gene expressions of p53 and p21, which down-regulated the expresseion of cyclinD1 and CDK2, and inhibited retinoblastoma protein (pRb) phosphorylation. This process could effectively arrest the cell cycle at G1 phase, which finally caused the inhibition on Neuro-2a cell proliferation. However, BDE-209 was only up-regulated the gene expressions of p53, also suggested to be involved in the inhibition on Neuro-2a cell proliferation.
\end{abstract}

Key words: 2,2'-,4,4'-tetrabromodiphenyl ether (BDE-47); decabrominateddiphenyl ether (BDE-209); Neuro-2a cells; cell proliferation; cell cycle; G1-phase arrest

\section{Introduction}

As new types of persistent organic pollutants (POPs), polybrominated diphenyl ethers (PBDEs) have been proved to possess high cytotoxicity such as inhibiting cell proliferation and inducing cellular apoptosis, which are reported in many kinds of human cells, such as 
hepatoma cell line (HepG2 cell) (Wang et al., 2012), T lymphocyte cells (Jurkat cell) (Yan et al., 2011) and various primary neurons or neuronal cell lines (He et al., 2008; Jiang et al., 2012). Many pathways are suggested to be involved in the process of cell proliferation inhibition induced by PBDEs stress, for instance, the detention of each phase in cell cycle, cell cycle phase arrest and cell death (including apoptosis and necrosis) (Evan and Vousden, 2001; King and Cidlowski, 1995), in which cell cycle arrest is the most acceptable one.

Cell cycle is a process that is strictly regulated by positive and negative cell cycle regulatory factors. Cyclin-dependent kinases (CDKs) are the core factors regulating the process by forming CDKs/cyclins complexes (Graña and Reddy, 1995; Boonstra, 2003). CDK inhibitors (CKIs), such as p21 and p27, are negative regulatory factors acting as the brakes of the cell cycle transition by preventing the formation of CDKs/Cyclin complexes (Collins and Garrett, 2005 ; Sherr and Roberts, 1999). Besides, the transition process is also regulated by a series of cell cycle checkpoints and restriction points, such as $\mathrm{R}$ point (Blagosklonny and Pardee, 2002), which is an essential checkpoint that is phosphorylated by CDKs/cyclins complexes and promotes the cell cycle moving from G1 to S phase (Weinberg, 1995). Therefore, these key proteins are often used to indicate the interference on cell cycle.

In fact, the alteration of cell cycle has a wide range of applications in the fields of toxicology and environmental monitoring. Kafi et al. (2013) had developed the electrochemical cell chip technique based on the principle of cell cycle to detect the environmental toxicants such as bisphenol-A (BPA) and poly chlorinated biphenyl (PCB). Song et al. (2015) reported quinone metabolite of PCB (PCB29-pQ) inhibited HepG2 cell proliferation through S-phase cell cycle arrest by down-regulating of cyclin A/D1/E and CDK 2/4/6 expression and the up-regulating of p21, p27 and p53 expression. Regarding to PBDEs, $\mathrm{Li}$ et al. (2012) reported PBDEs at low concentrations promoted the proliferations of $\mathrm{CHO}$ cell and OVCAR-3 cell through the induction of S phase and the elevation of G2/M phase, respectively. Wang et al. (2012) found BDE-47 promoted cell proliferation associated with the incresed expression of Cyclin D1 and the decrease in the G1 phase of cell cycle. We had reported in the previous studies that BDE-47 and BDE-209 exerted the impacts of 
cytotoxicity and apoptosis induction on Neuro-2a cells (Chen et al., 2015; Chen et al., 2016), and the cell proliferation was significantly inhibited by PBDEs exposure. What we focused in the present study was whether cell cycle arrest was involved in the observed results above mentioned? What was the possibly effective mechanism? The controlled experimental toxicological experiments were thus performed to bring light on the questions in the present study.

\section{Materials and methods}

\subsection{Cell culture and Reagents}

Murine neuroblastoma cell line (Neuro-2a cell, No. TCM29) was obtained from the Cell Bank of the Chinese Academy of Science (CAS, Shanghai, China). The cells were incubated in minimum essential medium (MEM) with $10 \%$ fetal bovine serum (FBS, $V / V), 100 \mathrm{U} / \mathrm{mL}$ penicillin and $100 \mu \mathrm{g} / \mathrm{mL}$ streptomycin (Gibco/Invitrogen Corp. Carlsbad, CA, USA). The cells were cultured in $5 \% \mathrm{CO}_{2}$ and a humidified incubator at $37^{\circ} \mathrm{C}$.

2,2',4,4'-tetrabromodiphenyl ether (BDE-47) was purchased from American AccuStandard Company (purity $>99.99 \%$, New Haven, USA) and Deca-BDE (BDE-209, 99.5\% purity) was provided by Dr. Ehrenstorfer Laboratories and purchased from Quandao Company (Shanghai, China). Dimethyl sulfoxide (DMSO) was used as the organic co-solvent (GC grade, Sigma, USA) in the present study. Both BDE-47 and BDE-209 were dissolved in DMSO to prepare the stock solution of $20 \mathrm{mM}$ and stored in dark at room temperature to avoid photolysis. The stock solution was diluted to the final working concentrations by adding an appropriate volume of DMSO to the culture medium. Based on our pre-experiments, the no-observed-effect concentration (NOEC) of DMSO for Neuro-2a cell was less than $0.5 \%$ $(V / V)$, thus the concentrations of DMSO in all the treatments were set less than $0.5 \%(V / V)$. Cell cycle detection Kit were purchased from Beyotime Biotechnology Inc. (Nantong, China). Trizol reagent, Reverse Transcript kit and SYBR PrimeScript RT-PCR Kit were purchased from TaKaRa (Dalian, China). All of the other chemicals and solvents were analytical or pharmaceutical grade. 


\subsection{Analysis on cell proliferation with and without PBDEs induction}

Eight concentrations of BDE-47 and BDE-209 were set in this experiment, which were 1, 5, 10, 20, 40, 60, 80 and $100 \mu \mathrm{M}$. Two controls were used in this experiment: a blank control of MEM medium without PBDEs addition, and a solvent control with DMSO $(0.5 \%, \mathrm{~V} / \mathrm{V})$. Neuro-2a cells were exposed to different treated groups for $48 \mathrm{~h}$, and MTT assay was applied to estimate the changes of cell proliferation according to the method of Ferrari et al. (1990). $10^{3}-10^{4}$ cells were seeded in a 96-well plate and were incubated overnight for the cells to adhere, then BDE-47 and BDE-209 at working concentrations were added into the cultures. Thereafter, the culture medium in the either treated groups or in the control were replaced by MTT solution $(500 \mu \mathrm{g} / \mathrm{mL}$; St. Louis, MO, USA). After $4 \mathrm{~h}$ incubation, the MTT formazan in cells were dissolved in $150 \mu \mathrm{L}$ DMSO. The absorbance was measured using a 2300 EnSpire ( $)$ Multilabel Reader (Perkin-Elmer, Turku, Finland) at $570 \mathrm{~nm}$. The change of cell proliferation was estimated according to the cells inhibition ratio, which was calculated as:

$$
\text { Inhibition ratio }(\%)=\frac{\mathrm{OD}_{\text {Control }}-\mathrm{OD}_{\text {Treament }}}{\mathrm{OD}_{\text {Control }}} \times 100 \%
$$

\subsection{Analysis on cell cycle with and without PBDEs induction}

Four concentrations of BDE-47 and BDE-209 were set in this experiment, which were 5, 10, 20, $40 \mu \mathrm{M}$. Two controls were used in this experiment: a blank control and a solvent control with DMSO $(0.2 \%, V / V)$. Neuro-2a cells were exposed to different treated groups for $48 \mathrm{~h}$, and cell cycle analysis in Neuro-2a cells was assessed using flow cytometry (FCM, Beckman Coulter, Cytomics FC 500 MPL, Fullerton, CA, USA) as described previously by Nunez et al. (2001). In brief, treated cells were harvested and fixed with $70 \%$ ethanol at $4{ }^{\circ} \mathrm{C}$ for $24 \mathrm{~h}$. The cell pellets were washed by PBS and resuspended in $1 \mathrm{~mL}$ of PBS containing $0.1 \mathrm{mg} / \mathrm{mL}$ RNase A at $37^{\circ} \mathrm{C}$ for $30 \mathrm{~min}$. Propidium iodide (PI, Beyotime Biotechnology Inc. Nantong, China) was added to the cell samples to a final concentration of about $50 \mu \mathrm{g} / \mathrm{mL}$, and then analyzed at FL-3 channel for PI fluorescence using FC 500 MPL flow cytometer (Beckman Coulter, CA, USA). The distribution of the cell cycle was indicated by the percentage of cells stained by PI in every phase. 


\subsection{Analysis on mRNA expression of G1-phase-relating regulatory factors using quantitative real-time PCR (qPCR)}

Two concentrations of BDE-47 and BDE-209 were set in this experiment, which were 20 and $40 \mu \mathrm{M}$, respectively. The controls setting were same as the experiment of cell cycle detection. a blank control and a negative control with DMSO $(0.2 \%, V / V)$. Neuro-2a cells were exposed to different treated groups for $48 \mathrm{~h}$, and the mRNA expression level of G1phase related regulatory factors $(\mathrm{CDK} 2, \mathrm{CDK} 4$, cyclin D1, cyclin E, p21 and p53) in Neuro2a cells were detected by qRT-PCR. Total RNA of all groups were extracted using the trizol reagent kit (TaKaRa, Dalian, China). The quality and the integrality of RNA were detected by A260/280 ratio and 1\% agarose gel, and then 500 ng RNA of all groups were reverse transcribed to cDNA using the reverse transcript kit (TaKaRa, Dalian, China). All primers sequences were listed in Table 1. The reaction buffer contained SYBR-Green, primers and cDNA template were amplification using an ABI 7500 QPCR Detection System (Applied Biosystems, CA, USA), and the amplification conditions was $95^{\circ} \mathrm{C}$ for $30 \mathrm{~s}$, followed by 40 cycles of $95^{\circ} \mathrm{C}$ for $5 \mathrm{~s}$, and $60{ }^{\circ} \mathrm{C}$ for $30 \mathrm{~s}$. Glyceraldehyde-3-phosphate dehydrogenase (GAPDH) was choose to be the housekeeping gene to normalize the expression levels of mRNA through the $2^{-\Delta \Delta C t}$ method (Livak and Schmittgen, 2001).

\subsection{Estimation on expression of pRb using western blot}

The treatment groups were the same as that described in 2.4. and the expression of $\mathrm{pRb}$ in Neuro-2a cells were evaluated by western blot. Cells in both treated groups and the controls were collected and lysed by RIPA lysis buffer. Protein was quantification by the BCA Protein Assay kit (Beyotime Bio. Inc., Nantong, China) and boiled for 8 min mixed with $5 \times$ SDS loading buffer. Proteins (40 $\mu \mathrm{g}$ per group) were separated using SDS-PAGE (8\% polyacrylamide gel) and transferred to PVDF membrane. The membrane was blocked by nonfat milk (5\%) for $2 \mathrm{~h}$ and then incubated with 1: 200 primary antibody of anti-pRb (1:200) goat polyclonal antibodies and anti- $\beta$-Actin (1: 500) rabbit polyclonal antibodies (Santa Cruz, CA, USA) overnight at $4{ }^{\circ} \mathrm{C}$. The secondary antibody conjugated horseradish peroxidase (HRP) was added for $2 \mathrm{~h}$ before visualization using ECL enhanced chemiluminescence (Pierce 
Biotechnology Inc., Rockford, IL, USA). $\beta$-actin was choose to be the housekeeping protein to normalize the expression levels of protein. The data were analyzed using Image-Pro Plus image analysis software (Media Cybernetics, Silver Spring, USA).

\subsection{Statistical analysis}

The data was obtained from at least three times independent experiments and presented as mean $\pm \mathrm{SD}$ (standard deviation). one-way analysis of variance (ANOVA) were used to identify difference between treated groups and solvent control. $P<0.05$ was considered to be statistical significance.

\section{Results}

\subsection{Effects of BDE-47 and BDE-209 on proliferation in Neuro-2a cells}

The results showed that both BDE-47 and BDE-209 significantly inhibited the cell proliferation compared to the DMSO control, but the ranges of effective concentrations were different: BDE-47 at a range from $20-100 \mu \mathrm{M}$ greatly inhibited the cell proliferation and the peak of inhibition ratio appeared at $100 \mu \mathrm{M}$ group, which was $(65.45 \pm 4.32) \%$ as compared to the control. A clear concentration-depend manner was observed between the concentration and the inhibitory ratio. Similarly, the inhibitory ratio increased steadily with the concentration increment in BDE-209 exposure groups, in which significance was observed when the stressing concentration was above $40 \mu \mathrm{M}$, and the peak of inhibition appeared in group of $100 \mu \mathrm{M}$, which was about (37.13 \pm 5.12$) \%$ (Fig.1). Results suggested that both BDE47 and BDE-209 inhibited the cell proliferation of Neuro-2a, and BDE-47 was more effect than BDE-209.

\subsection{Effect of BDE-47 and BDE-209 on the changes of cell cycle in Neuro-2a cells}

The results of flow cytometry (FCM) showed that BDE-47 elevated the percentage of cells in the G0/G1 phase but decreased the percentage of cells in the $\mathrm{S}$ phase. There was slightly changes happened to the percentage of cells in the G2/M phase compared with the DMSO control (Fig. 2). To be specific, the proportion of cells in the G0/G1 phase in the group treated with $40 \mu \mathrm{M}$ BDE-47 was $(56.24 \pm 1.38) \%$, which was significantly higher than 
that in the DMSO control group $(37.29 \pm 1.91) \%(P<0.05)$, whereas the proportion of cells in the S phase was $(41.73 \pm 0.92) \%$, which was significantly lower than that found in the DMSO control $(20.97 \pm 2.04) \%(P<0.05)$. The stress of BDE-209 slightly elevated the percentage of cells in the G0/G1 phase but not statistical significant $(P>0.05)$, but failed to induce the changes in the percentage of cells in the $\mathrm{S}$ phase and G2/M phase $(P>0.05)$. Thus, BDE-47 might cause G1 phase cell cycle arrest though increasing the percentage of cells in the G0/G1 phase and decreasing the percentage of cells in the S phase, while BDE-209 had no obviously change in cell cycle.

\subsection{Effects of BDE-47 and BDE-209 on the changes of G1-phase-relating regulatory factors in Neuro-2a cells}

The mRNA expression levels of cyclin D1 and CDK2 were significantly decreased after exposure to BDE-47 $(P<0.05)$ (Fig. 3a,c), and the mRNA expression level of CDK4 was slightly decreased $(P<0.05)$, while there was no obviously change in cyclin E2 mRNA expression level $(P>0.05)$ (Fig. 3b,d). However, exposure to BDE-209 failed to make a significantly change in these genes expression $(P>0.05)$ (Fig. 3). Thereby, BDE-47-mediated cell cycle arrest in Neuro-2a cells was correlated with a down-regulated of the expressions of cyclin D1 and CDK 2.

The mRNA level of p21 was slightly increased when exposure to $40 \mu \mathrm{M}$ BDE-47 and BDE-209 (Fig. 3e). The p53 mRNA expression was largely increased in both BDE-47 and BDE-209 treated cells $(P<0.05)$ (Fig. 3f). mRNA expression level of p53 were increased (4.10 \pm 0.22$)$ and (1.23 \pm 0.03$)$ folds in $40 \mu \mathrm{M}$ BDE-47 and BDE-209 treatment group compared with DMSO control.

The degree of phosphorylation of $\mathrm{pRb}$ in Neuro-2a cells was reduced showed by western blot after treated with BDE-47 and BDE-209. Phosphorylation of pRb exhibited a weaker immunoreactive band in $40 \mu \mathrm{M}$ BDE-47 treatment group compared with DMSO control, and quantification analysis indicated the expression level phosphorylated of $\mathrm{pRb}$ was significantly decreased $(P<0.05)$ (Fig. 4). BDE-209 treatment in the same concentration had a significantly 
effect on this protein. Results indicated BDE-47 induced cell cycle arrest in Neuro-2a cells was associated with the inhibition of $\mathrm{pRb}$ phosphorylation.

\section{Discussion}

PBDEs induced cytotoxicity in vitro is mainly indicated by the inhibition of cell proliferation and the induction of apoptosis (He et al., 2008; Li et al., 2012; Souza et al., 2013). Apoptosis and cell cycle abnormal might be responsible for the inhibition of cell proliferation. The possible mechanism of apoptosis induced by PBDEs have been well studied on the former research reports and our preliminary studies (Chen, 2015; Costa et al., 2014; Yan et al., 2011; Zhang et al., 2013), thus our present study foucused on the effects of BDE47 and BDE-209 on the cell cycle in Neuro-2a cells and their possible mechanisms. BDE-47 and BDE-209 inhibited Neuro-2a cells proliferation in a concentration dependent manner, and inhibited rates increased significantly above $20 \mu \mathrm{M}$ of BDE-47 and $40 \mu \mathrm{M}$ of BDE-209 exposure at $48 \mathrm{~h}$ (Fig. 1), indicating Neuro-2a cells were more sentitive to BDE-47 than BDE-209. BDE-47 caused inhibition of proliferation were also found in other non-neuron cell lines and neuron cell lines, such as Jurkat T cells (Yan et al., 2011), macrophages (Lv et al., 2015), RTG-2 cells (Jin et al., 2010), SH-SY5Y cells (Jiang et al., 2012) and primary rat hippocampal neuron (He et al., 2008). Both previous studies and present study showed BDE47 had higher cytotoxicity than BDE-209, which was consistent with the finding that higherbrominated congeners are less toxic than the lower-brominated congeners (Jin et al., 2010). The effect on cell proliferation is related to the exposure concentrations of PBDEs. Li et al. (2012) reported that BDE-209 promoted proliferation of germ cells at low concentrations. Wang et al. (2012) reported a hormesis effect of BDE-47 on HepG2 cells that the cell proliferation was promoted by low concentrations and inhibited by high concentrations. We considered high concentrations of PBDEs are better for studying cell proliferation inhibition and related mechanism, although the range concentrations may higher than that detected in the environmental or human exposure. Based on this, we set up our experimental concentrations of PBDEs range from 10 to $40 \mu \mathrm{mol} / \mathrm{L}$ for cell cycle analysis in our study. 
Cell proliferation inhibition is usually associated with abnormal progression of the cell cycle (King and Cidlowski, 1995). Flow cytometric analysis was performed to determine whether cell proliferation inhibited by the two PBDEs are accompanied with cell cycle arrest. The result showed that treatment with BDE-47 $(20$ and $40 \mu \mathrm{M})$ increased the percentage of cells in G0/G1 phase and decreased the percentage of cells in the $S$ phase (Fig. 2), indicating that the cell cycle progression may be hampered in G1 phase. However, treated with BDE209 only showed a slight change on cell cycle distribution. This result indicated that BDE-47 prevented the cell cycle progression from G1 to S phase and contributed to at least partially the inhibition of cell proliferation. Li et al. (2012) claimed BDE-209 promoted proliferation of germ cells, which mediated through increasing the propotions of cells in the S phase and the $\mathrm{G} 2 / \mathrm{M}$ phase, respectively.

Cell cycle is regulated by an intricate network involved in positive regulatory factors including cyclins and CDKs, negative regulatory factors like CKIs, and the checkpoints among different phases (Graña and Reddy, 1995). BDE-47 suppressed Neuro-2a cells proliferation through arresting cell cycle at G1 phase, therefore related regulatory factors of G1 phase were detected. The progression of G1 phase and G1/S phase transition are controlled by CDK4-cyclin D and CDK2-cyclin E complexes (Boonstra, 2003). Here, BDE47 obviously decreased the mRNA expression of CDK2, and slightly reduced CDK4 expression. In addition, the expression levels of cyclin D1 were decreased by BDE-47, but there was no visible change in cyclin E2 expression (Fig. 3), indicating both the CDK4-cyclin $\mathrm{D}$ and CDK2-cyclin E complexes are inhibited. $\mathrm{pRb}$ is the checkpoint of G1 cell cycle progression, which could be phosphorylated by CDK2-cyclin E and CDK4-cyclin D complexes, and its phosphorylation blocked could inhibit G1-S phase transition (Bartek et al., 1996; Weinberg, 1995). The result showed that BDE-47 reduced expression of phosphorylated pRb (Fig. 4), indicating inhibited the combined of CDK2-cyclin E and CDK4-cyclin D complexes leading to suppressed the phosphorylation of $\mathrm{pRb}$. However, exposure to BDE-209 did not cause significantly changes on these regulatory factors. Both BDE-47 and BDE-209 up-regulated the mRNA expression of p21 in Neuro-2a cells but did 
not reach the significance range $(P>0.05)$ (Fig. 3e), which might be the result of G1 arrest by inhibiting the activities of CDK2 and cyclin D and disturbing the combination of CDK2cyclin E and CDK4-cyclin D complexes. p21 is mainly regulated by the p53 tumor suppressor protein (Waldman et al., 1995). BDE-47 up-regulated p53 mRNA expression, which could increase the downstream gene p21 expression and inhibit cell cycle progression. BDE-209 also up-regulated p53 mRNA expression, but did not cause obvious cell cycle arrest as BDE47. It has been reported PCB29-pQ promoted p53-dependent cycle arrest and apoptosis in HepG2 cells, in which p53 played a central role in this metabolite-induced cell cycle arrest and apoptosis, thus we considered p53 might be the central molecule in the cross-talk of cell PBDEs leading to cycle arrest and apoptotic signaling.

In conclusion, BDE-47 and BDE-209 inhibited Neuro-2a cells proliferation, and BDE-47 was more potent than BDE-209. BDE-47 might promote p53-mediated G1-phase arrest, which was supported by the following results: (a) BDE-47 increased the expression of p53 and p21 which inhibited CDK2 activity; (b) BDE-47 reduced the expression of cyclin D1 and $\mathrm{CDK} 2$, preventing the combination of CDK4/cyclin D and CDK2/cyclin E complexes; (c) BDE-47 inhibited the phosphorylation of $\mathrm{pRb}$, and eventually arrested cells cycle prossession. BDE-209 increased the expression of p53, also suggested to be involved in the inhibition of cell proliferation, but the effect was not so obvious as that of BDE-47.

\section{Conclusions}

When combined considered the results in the present study we concluded that: both BDE-47 and BDE-209 inhibited Neuro-2a cells proliferation, and BDE-47 was more effective than BDE-209. BDE-47 promoted p53-mediated G1-phase arrest involved in stimulating the expression of p21 and p53 and suppressed the expression of CDK 2 and Cyclin D, which resulted in inhibited phosphorylation of $\mathrm{pRb}$, and eventually prevented the process of cell cycle from G1 phase to S phase. BDE-209 increased p53 expression and inhibited Neuro-2a cells proliferation, but had a slightly effect on cell cycle. The data provided in this study gave 
a better understanding on the mechanism underlying the anti-proliferative effect of BDE-47 and BDE-209 on Neuro-2a cells.

\section{Acknowledgments}

This project was supported by the National Natural Science Foundation of China (No. 41476109 and No. 41276140) and the Joint Fund of the National Science Foundation of China and Shandong Province (Grant No. NSFC-U 1406403). We are grateful to Dr. Yan Zhao at Ocean University of China for the guidance on the manuscript.

\section{References}

Bartek, J., Bartkova, J., Lukas, J., 1996. The retinoblastoma protein pathway and the restriction point. Curr. Opin. Cell Biol. 8, 805-814.

Blagosklonny, M.V., Pardee, A.B., 2002. The restriction point of the cell cycle. Cell Cycle 1, 102-109.

Boonstra, J., 2003. Progression through the G1-phase of the on-going cell cycle. J. Cell Biochem. 90, 244-252.

Chen, H., Tang, X., Zhou, B., Xu, N., Sha, J., Guo, H. et al, 2015. Cytotoxicity and apoptosis induction in Neuro-2a cells of BDE-47 and BDE-209. WIT Transactions on Biomedicine and Health 7.

Chen, H., Tang, X., Zhou, B., Xu, N., Wang Y., 2016. Mechanism of Deca-BDE-induced apoptosis in Neuro-2a cells: Role of death-receptor pathway and reactive oxygen species-mediated mitochondrial pathway. J. Environ. Sci. (China) 46, 241-251.

Collins, I., Garrett, M.D., 2005. Targeting the cell division cycle in cancer: CDK and cell cycle checkpoint kinase inhibitors. Curr. Opin. Pharmacol. 5, 366-373.

Costa, L.G., de Laat, R., Tagliaferri, S., Pellacani, C., 2014. A mechanistic view of polybrominated diphenyl ether (PBDE) developmental neurotoxicity. Toxicol. Lett. 230, 282-294.

Evan, G.I., Vousden, K.H., 2001. Proliferation, cell cycle and apoptosis in cancer. Nature 411, $342-348$.

Ferrari, M., Fornasiero, M.C., Isetta, A.M., 1990. MTT colorimetric assay for testing macrophage cytotoxic activity in vitro. J. Immunol. Methods 131(2): 165-172. 
Graña, X., Reddy, E.P., 1995. Cell cycle control in mammalian cells: role of cyclins, cyclin dependent kinases (CDKs), growth suppressor genes and cyclin-dependent kinase inhibitors (CKIs). Oncogene 11, 211-219.

He, P., He, W., Wang, A., Xia, T., Xu, B., Zhang, M. et al, 2008. PBDE-47-induced oxidative stress, DNA damage and apoptosis in primary cultured rat hippocampal neurons. Neurotoxicology 29, 124-129.

Jiang, C., Zhang, S., Liu, H., Zeng, Q., Xia, T., Chen, Y. et al, 2012. The role of the IRE1 pathway in PBDE-47-induced toxicity in human neuroblastoma SH-SY5Y cells in vitro. Toxicol. Lett. 211, 325-333.

Jin, S., Yang, F., Hui, Y., Xu, Y., Lu, Y., Liu, J., 2010. Cytotoxicity and apoptosis induction on RTG-2 cells of 2, 2', 4, 4'-tetrabromodiphenyl ether (BDE-47) and decabrominated diphenyl ether (BDE-209). Toxicol. in Vitro 24, 1190-1196.

Kafi, M.A., Yea, C.H., Kim, T.H., Yagati, A.K., Choi, J.W., 2013. Electrochemical cell chip to detect environmental toxicants based on cell cycle arrest technique. Biosens. Bioelectron. 41, 192-198.

King, K.L., Cidlowski, J.A., 1995. Cell cycle and apoptosis: common pathways to life and death. J. Cell Biochem. 58, 175-180.

Li, Z.H., Liu, X.Y., Wang, N., Chen, J.S., Chen, Y.H., Huang, J.T. et al, 2012. Effects of decabrominated diphenyl ether (PBDE-209) in regulation of growth and apoptosis of breast, ovarian, and cervical cancer cells. Environ. Health Persp. 120, 541-546.

Livak, K.J. and Schmittgen, T.D., 2001. Analysis of relative gene expression data using realtime quantitative PCR and the 2(-Delta Delta C(T)) Method. Methods 25: 402-408.

Lv, Q., Wan, B., Guo, L., Zhao, L., Yang, Y., 2015. In vitro immune toxicity of polybrominated diphenyl ethers on murine peritoneal macrophages: Apoptosis and immune cell dysfunction. Chemosphere 120, 621-630.

Nunez, R., 2001 DNA measurement and cell cycle analysis by flow cytometry. Curr. Issues. Mol. Boil. 3: 67-70.

Sherr, C.J., Roberts, J.M., 1999. CDK inhibitors: positive and negative regulators of G1-phase progression. Gene Dev. 13, 1501-1512.

Song, X., Li, L., Shi, Q., Lehmler, H.J., Fu, J., Su, C. et al, 2015. Polychlorinated Biphenyl Quinone Metabolite Promotes p53-Dependent DNA Damage Checkpoint Activation, SPhase Cycle Arrest and Extrinsic Apoptosis in Human Liver Hepatocellular Carcinoma 
HepG2 Cells. Chem. Res. Toxicol. 28, 2160-2169.

Souza, A.O., Pereira, L.C., Oliveira, D.P., Dorta, D.J., 2013. BDE-99 congener induces cell death by apoptosis of human hepatoblastoma cell line-HepG2. Toxicol. in Vitro 27, $580-587$.

Waldman, T., Kinzler, K.W., Vogelstein, B., 1995. p21 is necessary for the p53-mediated G1 arrest in human cancer cells. Cancer Res. 55, 5187-5190.

Wang, L., Zou, W., Zhong, Y., An, J., Zhang, X., Wu, M. et al, 2012. The hormesis effect of BDE-47 in HepG2 cells and the potential molecular mechanism. Toxicol. Lett. 209, 193201.

Weinberg, R.A., 1995. The retinoblastoma protein and cell cycle control. Cell 81, 323-330.

Yan, C., Huang, D., Zhang, Y., 2011. The involvement of ROS overproduction and mitochondrial dysfunction in PBDE-47-induced apoptosis on Jurkat cells. Exp. Toxicol. Pathol. 63, 413-417.

Zhang, S., Kuang, G., Zhao, G., Wu, X., Zhang, C., Lei, R. et al, 2013. Involvement of the mitochondrial p53 pathway in PBDE-47-induced SH-SY5Y cells apoptosis and its underlying activation mechanism. Food Chem. Toxicol. 62, 699-706.

\section{Figure captions}

Figure 1. Analysis of cell inhibit rate changes in Neuro-2a cells exposed to different concentrations of BDE-47 and BDE-209. "Indicated the significance at $P<0.05$ level and ***indicated the most significant at $P<0.01$ level. Data were expressed as means $\pm \operatorname{SD}(n=3)$.

Figure 2. Cell cycle analysis of Neuro-2a cells after exposed to different concentrations of BDE-47 and BDE-209. (a) Representative graphs of cell cycle distribution obtained from flow cytometry analysis; percentage of cells in cycle phase distribution were shown in histogram when treated with BDE-47 (b) and BDE-209 (c). * Indicated the significant difference between the treated groups with the control group at the $P<0.05$ level, and data were expressed as means $\pm \operatorname{SD}(n=3)$.

Figure 3. Changes of mRNA expression of G1-phase-relating regulatory genes in Neuro-2a cells exposed to different concentrations of BDE-47 and BDE-209. The mRNA expression of Cyclin D1 (a), Cyclin E2 (b), CDK2 (c), CDK4 (d), p21 (e) and p53 (f). *Indicated the 
significant difference between the treated groups with the DMSO control group at the $P<$ 0.05 level and ${ }^{* *}$ indicated the most significant at $P<0.01$ level, and data were expressed as means \pm SD $(n=3)$.

Figure 4. Western blot analysis on the phosphorylation of pRb in Neuro-2a cells exposed to different concentrations of BDE-47 and BDE-209. (a) Representative autoradiographs of phosphorylation of $\mathrm{pRb}$ expression, $\beta$-actin was used to confirm the equal loading of proteins; (b) the mean density value of phosphorylation of $\mathrm{pRb}$ was expressed as a ratio relative to that of $\beta$-actin. *Indicated the significance difference between the treated groups with the DMSO control group at $P<0.05$ level and ${ }^{* *}$ indicated the most significant at $P<0.01$ level, and data were expressed as means $\pm \operatorname{SD}(n=3)$.

Figure 5. Possible mechanism of BDE-47 and BDE-209 induced cell cycle arrest in Neuro-2a cells.

\section{List of tables}

\begin{tabular}{llll}
\hline Jenes & Genebank & Primer sequence & Size (bp) \\
\hline p53 & NM_011640.3 & Forward: 5'-GGGCTGAGACACAATCCTC-3' & 176 \\
& & Reverse: 5'-TAGGTGCCAGGGTCCAAC-3' & \\
p21 & NM_001111099.1 & Forward: 5'-CAGGGATGGCAGTTAGGACT-3' & 132 \\
& & Reverse: 5'-AGAGACCCACAGGAGAGGTG-3' & \\
cydin D1 & NM_007631.2 & Forward: 5'-CAGAAGTGCGAAGAGGAGGT-3' & 152 \\
& & Reverse: 5'-GCAGTCAAGGGAATGGTCTC-3' & \\
cydin E2 & NM_001037134.2 & Forward: 5'-TCTGTGCATTCTAGCCATCG-3' & 118 \\
& & Reverse: 5'-ATCCCATTCCAAACCTGAAG-3' & \\
CDK4 & NM_009870.3 & Forward: 5'-GATTGCCTCCAGAAGACGAC-3' & 115 \\
& & Reverse: 5'-GCTCCAGACTCCTCCATCTCT-3' & \\
& & Forward: 5'-GGTCTGTTCATCGTGGTTCA-3' & 101 \\
CDK2 & NM_016756.4 & Reverse: 5'-AAGTCCTGGGTGTAGCAGTCA-3' & \\
& & Forward: 5'-TGTGTCCGTCGTGGATCTGA-3' & 123
\end{tabular}




\section{Reverse: 5'-TTGCTGTTGAAGTCGCAGGAG-3'}

Table 1 Primer sequences of the analyzed target genes and product length (bp). 


\section{List of figures}

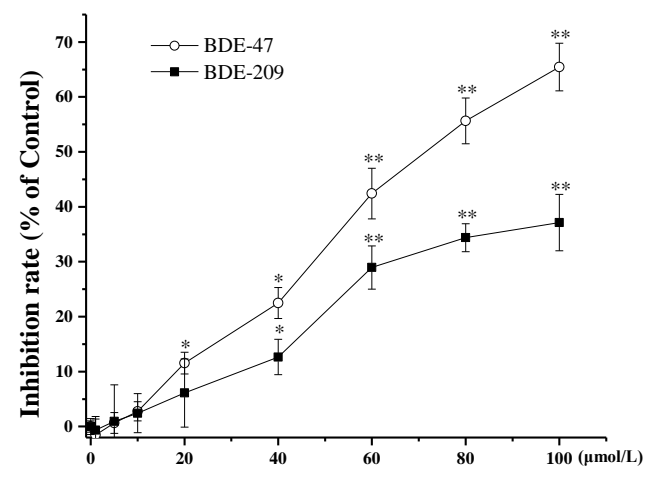

Figure 1

a
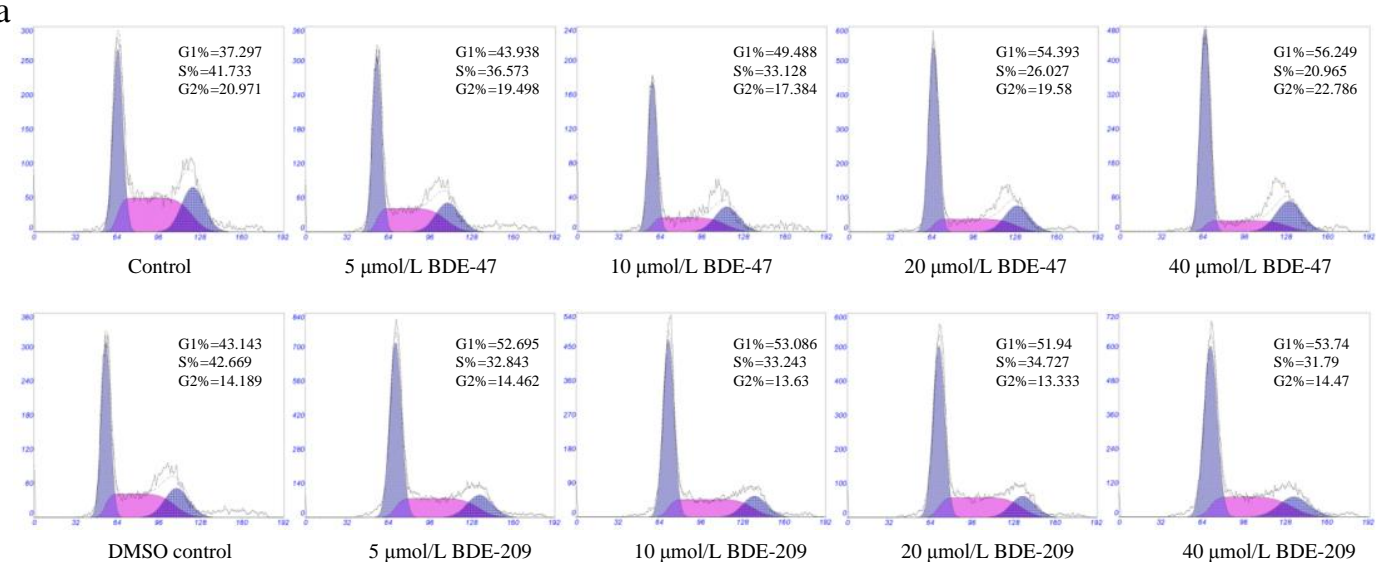

b

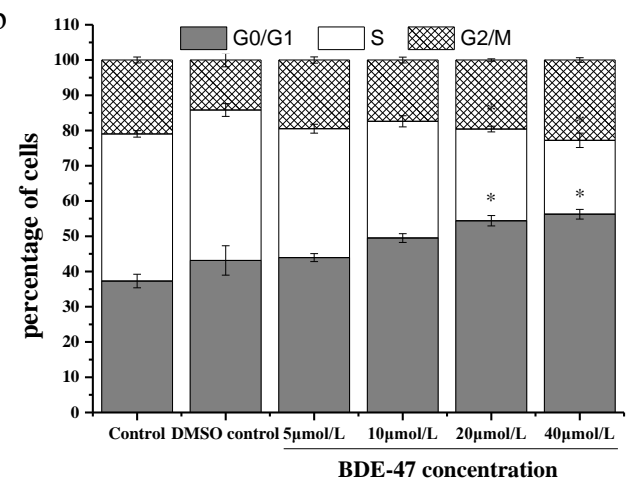

c

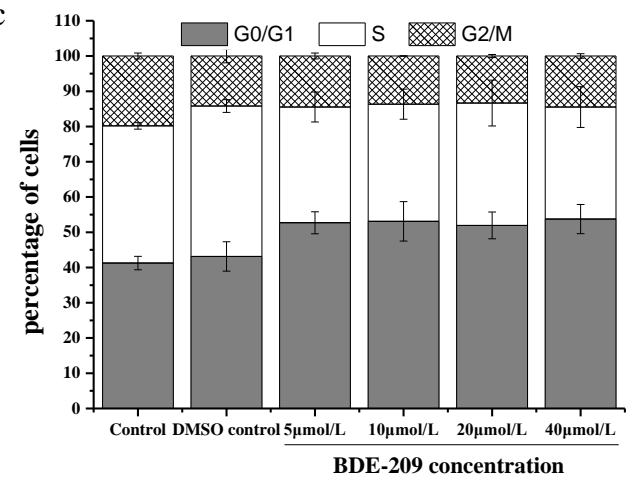

Figure 2 

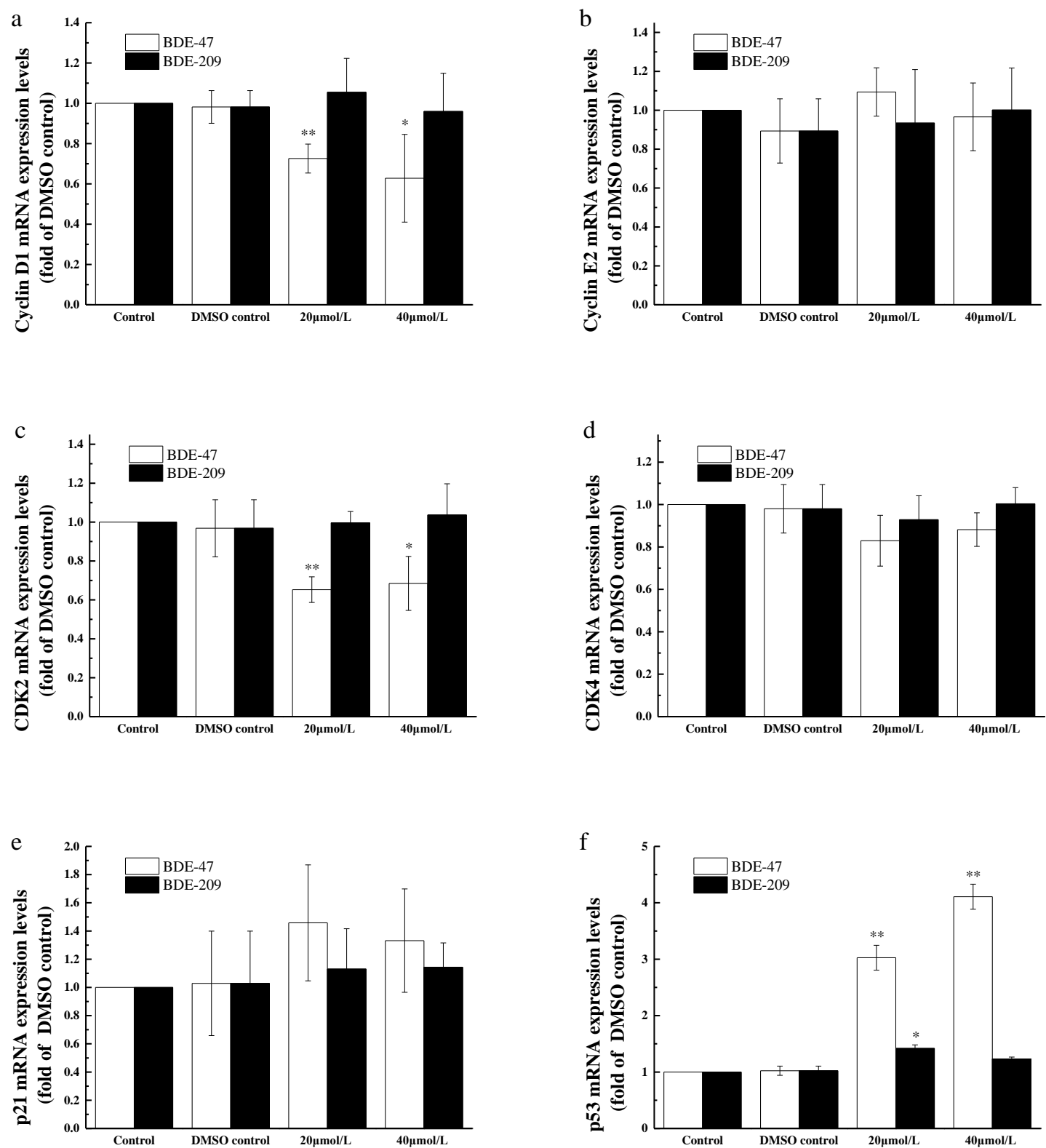

Figure 3
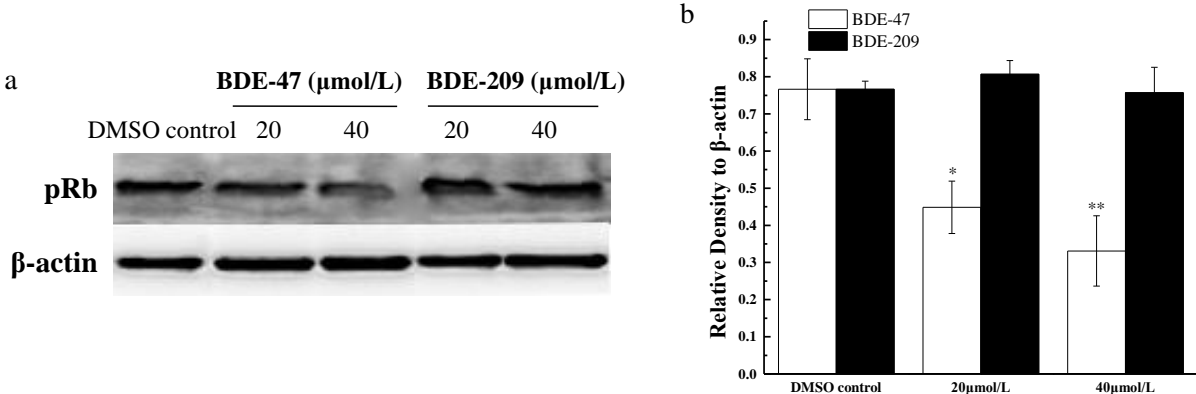

Figure 4 


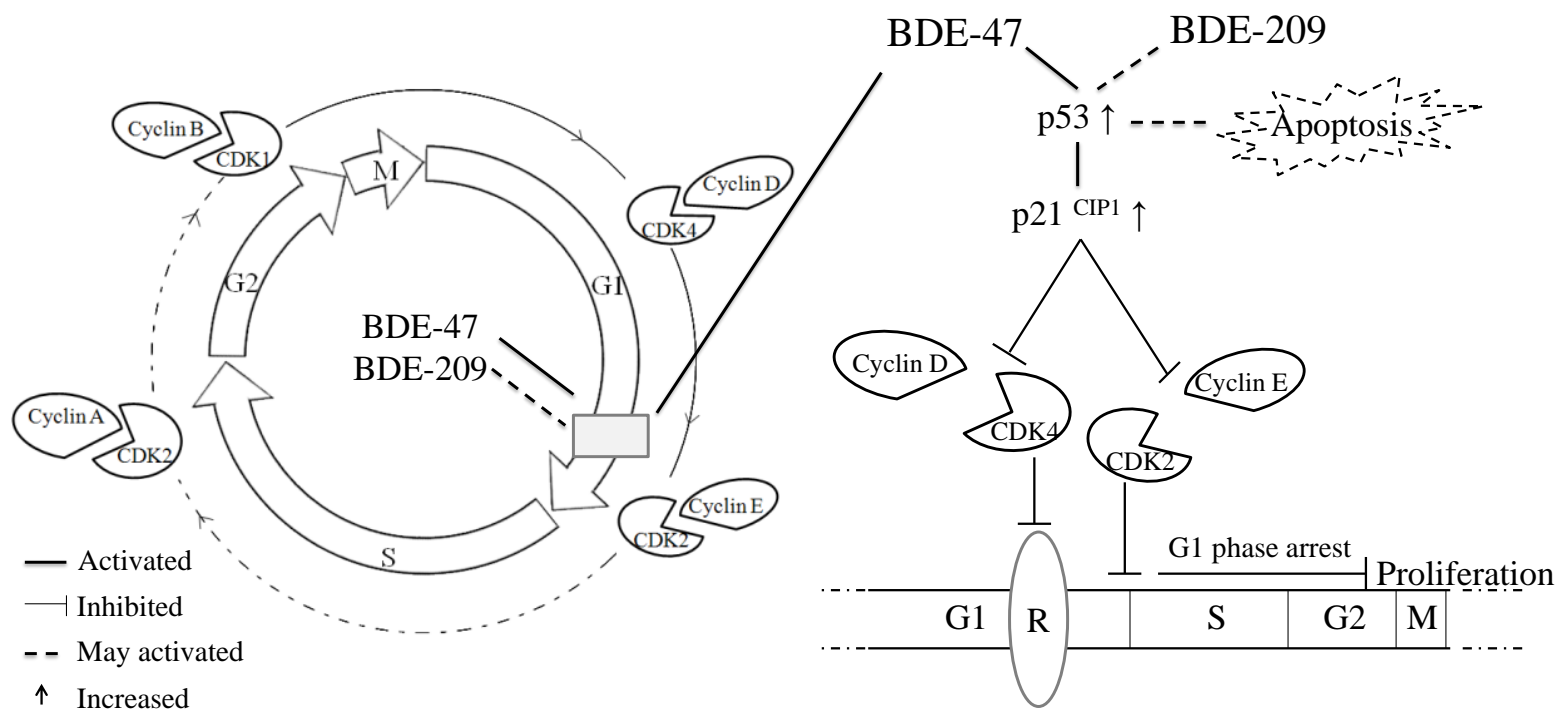

Figure 5 